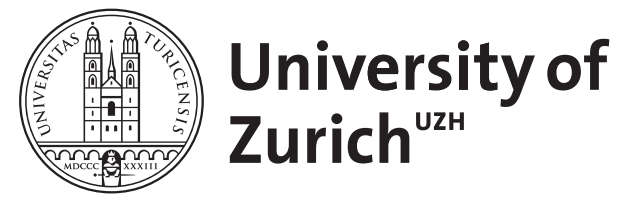
Archive

University of Zurich

University Library

Strickhofstrasse 39

CH-8057 Zurich

www.zora.uzh.ch

Year: 2013

Nonlocal van der Waals functionals: The case of rare-gas dimers and solids

Tran, Fabien ; Hutter, Juerg

DOI: https://doi.org/10.1063/1.4807332

Posted at the Zurich Open Repository and Archive, University of Zurich

ZORA URL: https://doi.org/10.5167/uzh-80279

Journal Article

Accepted Version

Originally published at:

Tran, Fabien; Hutter, Juerg (2013). Nonlocal van der Waals functionals: The case of rare-gas dimers and solids. Journal of Chemical Physics, 139(3):204103.

DOI: https://doi.org/10.1063/1.4807332 


\section{Nonlocal van der Waals functionals: The case of rare-gas dimers and solids}

Fabien $\operatorname{Tran}^{1, \text { a) }}$ and Jürg Hutter ${ }^{1}$

Institute of Physical Chemistry, University of Zurich, Winterthurerstrasse 190, CH-8057 Zurich, Switzerland

(Dated: 2 May 2013)

Recently, the nonlocal van der Waals (vdW) density functionals [Dion et al., Phys. Rev. Lett. 92, 246401 (2004)] have attracted considerable attention due to their good performance for systems where weak interactions are important. Since the physics of dispersion is included in these functionals, they are usually more accurate and show less erratic behavior than the semilocal and hybrid methods. In this work, several variants of the vdW functionals have been tested on rare-gas dimers (from $\mathrm{He}_{2}$ to $\mathrm{Kr}_{2}$ ) and solids (Ne, Ar, and $\mathrm{Kr}$ ) and their accuracy compared to standard semilocal approximations supplemented or not by an atom-pairwise dispersion correction [Grimme et al., J. Chem. Phys. 132, 154104 (2010)]. An analysis of the results in terms of energy decomposition is also provided.

a)f.tran@pci.uzh.ch 


\section{INTRODUCTION}

Thanks to its relatively low cost/accuracy ratio, the Kohn-Sham (KS) ${ }^{1}$ version of density functional theory $(\mathrm{DFT})^{2}$ is the most used quantum method for the calculation of the geometrical and electronic properties of molecules, surfaces, and solids. The accuracy of the results of a KS-DFT calculation depends primarily on the chosen approximation for the exchange-correlation functional (xc) $E_{\mathrm{xc}}$ (see Ref. 3 for a recent review). Nowadays, the most popular types of approximations for $E_{\mathrm{xc}}$ are the semilocal [in particular the generalized gradient approximation (GGA) ${ }^{4,5}$ ] and hybrid functionals, ${ }^{6}$ which very often give satisfactory results. However, it is well known that by construction none of these two approximations account properly for the dispersion interactions, which arise due to the attraction between non-permanent dipoles, and that the results obtained with semilocal and hybrid methods on systems where dispersion interactions play a major role are often unreliable (see, e.g., Refs. 7 and 8).

Therefore, efforts have been made to propose methods within the framework of KS-DFT which explicitly account for the dispersion interactions (see Refs. 9-12 for reviews). Among these methods, the simplest consist of adding to the KS-DFT total energy a dispersion term of the form

$$
E_{\text {disp }}=-\sum_{A<B} \sum_{n=6,8,10, \ldots} f_{n}^{\text {damp }}\left(R_{A B}\right) \frac{C_{n}^{A B}}{R_{A B}^{n}},
$$

where $C_{n}^{A B}$ are the dispersion coefficients for the atom pair $A$ and $B$ separated by the

distance $R_{A B}$ and $f_{n}^{\text {damp }}$ is a damping function preventing Eq. (1) to become too large at small $R_{A B}$. The coefficients $C_{n}^{A B}$ can be either precomputed (see, e.g., Refs. 13-15) or calculated using properties (e.g., electron density) of the system under consideration like in the exchange-hole dipole moment model (XDM) of Becke and Johnson ${ }^{16}$ or the method of Tkatchenko and Scheffler. ${ }^{17}$ The DFT-D2 ${ }^{15}$ and DFT-D3 ${ }^{18}$ versions of Grimme are currently the most widely used of these methods. One of the advantages of most methods using Eq. (1) is to add a relatively negligible computational cost compared to the calculation of the KS-DFT energy.

Another group of methods accounting explicitly of dispersion interactions consist of adding a nonlocal term of the form

$$
E_{\mathrm{c}}^{\mathrm{nl}}=\frac{1}{2} \iint \rho(\mathbf{r}) \Phi\left(\mathbf{r}, \mathbf{r}^{\prime}\right) \rho\left(\mathbf{r}^{\prime}\right) d^{3} r d^{3} r^{\prime}
$$


to a LDA (local density approximation) or GGA correlation functional. In Eq. (2), the kernel $\Phi$ depends on quantities at $\mathbf{r}$ and $\mathbf{r}^{\prime}$ :

$$
\Phi\left(\mathbf{r}, \mathbf{r}^{\prime}\right)=\Phi\left(\rho(\mathbf{r}), \rho\left(\mathbf{r}^{\prime}\right),|\nabla \rho(\mathbf{r})|,\left|\nabla \rho\left(\mathbf{r}^{\prime}\right)\right|,\left|\mathbf{r}-\mathbf{r}^{\prime}\right|\right)
$$

The first functional of the form given by Eq. (2), which could be applied to any type of systems was proposed by Dion et al. (DRSLL) ${ }^{19}$ The DRSLL term was derived starting from the adiabatic connection-fluctuation dissipation theorem. ${ }^{20-22}$ Originally, it was used in combination with revPBE ${ }^{23}$ for exchange and LDA for correlation and the functional is named as vdW-DF in the literature (in Table I the composition of the functionals tested in the present work are given). vdW-DF was shown to be a clear improvement over the commonly used functionals, however it became also obvious that a serious shortcoming of $\mathrm{vdW}-\mathrm{DF}$ is to systematically overestimate the equilibrium distances. ${ }^{24,25}$

Therefore, several attempts have been made to remedy this problem by combining the DRSLL nonlocal term with a more compatible semilocal functional or by proposing a new nonlocal term. For instance, Lee et al. (LMKLL) ${ }^{26}$ proposed to modify slightly the DRSLL term (by changing the value of one parameter) and to use it in combination with PW86R ${ }^{27}$ (a refitted version of the Perdew-Wang functional PW86 ${ }^{28}$ ). Their resulting functional (called vdW-DF2) was shown to improve over the original vdW-DF. In Ref. 29, a new

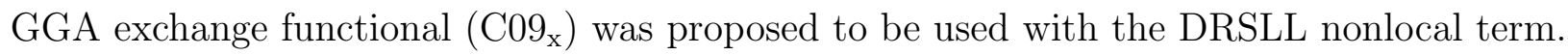
The functional, $\mathrm{C} 09_{\mathrm{x}} \mathrm{vdW}$, leads to more accurate results than vdW-DF for various types of systems. In Refs. 30 and 25, Klimeš et al. combined many GGA exchange functionals (already existing or newly proposed) with the DRSLL term. Particularly interesting are the functionals optB88-vdW and optPBE-vdW which lead to accurate results for both finite ${ }^{30}$ and extended ${ }^{25}$ systems. We also mention Ref. 31, where the nonlocal DRSLL term is used with the GGA RPBE exchange functional ${ }^{32}$ and with either LDA for correlation or a linear combination of the LDA and PBE correlation functionals, the latter case leading to the functional named RPBEc2 $/ 3+$ nl (see Table I).

Vydrov and Van Voorhis proposed their own nonlocal functionals, VV09 ${ }^{35}$ and VV10 ${ }^{36}$ [also of the form given by Eq. (2)], which were constructed such that also the short-range regime of the van der Waals interactions is adequately described. VV10, when added to PW86R ${ }^{27}$ exchange and PBE correlation, has been shown to be particularly accurate for finite systems (see, e.g., Ref. 37). However, its performance for solids is rather bad, ${ }^{38,39}$ 
TABLE I. Composition of the tested exchange-correlation functionals. The VWN5 parametrization $^{33}$ is used for LDA correlation.

\begin{tabular}{lccc}
\hline \hline Functional & Reference Exchange & Correlation \\
\hline LDA & 1 and 33 & LDA & LDA \\
PBE & 5 & PBE & PBE \\
vdW-DF & 19 & revPBE & LDA+DRSLL \\
vdW-DF2 & 26 & PW86R & LDA+LMKLL \\
C09 -vdW & 29 & C09 & LDA+DRSLL \\
optB88-vdW & 30 & optB88 & LDA+DRSLL \\
RPBEc2/3+nl & 31 & RPBE & $\frac{1}{3}$ LDA+ $\frac{2}{3}$ PBE+DRSLL \\
rVV10 & 34 & PW86R & PBE+rVV10 \\
PBE-D3 & 18 & PBE & PBE+D3 \\
revPBE-D3 & 18 & revPBE & PBE+D3 \\
B97D-D3 & 18 & B97D & B97D+D3 \\
\hline \hline
\end{tabular}

therefore two parameters of the VV10 functional were modified such that the results for solids are improved. ${ }^{39}$

In this work, the results obtained for the equilibrium distance and interaction energy of rare-gas dimers and solids will be presented. The focus will be on the performance of several nonlocal functionals (listed in Table I) with which only a few calculations on rare-gas systems have been done up to now. The rare-gas systems are the prototypical van der Waals systems where the dispersion interactions are the only source of attraction between atoms and for which highly accurate $a b$ initio or empirical results are available. The rare-gas dimers have been used numerous times for the testing of functionals for weak interactions (see, e.g., Refs. 40-53 for extensive tests), while tests on rare-gas solids are less common and recent. ${ }^{54-62}$

\section{METHODS}

The calculations were done with the Quickstep module ${ }^{63}$ of the CP2K program package, ${ }^{64}$ which is based on a mixed Gaussian and plane waves formalism. ${ }^{65}$ More specifically, we used the Gaussian and augmented-plane-wave method (GAPW), ${ }^{66}$ which allows for all-electron 
calculations. The calculations on the rare-gas dimers $\mathrm{He}_{2}, \mathrm{Ne}_{2}, \mathrm{Ar}_{2}$, and $\mathrm{Kr}_{2}$ were done with the augmented correlation consistent polarized quintuple zeta (aug-cc-pV5Z) basis sets, ${ }^{67,68}$ which lead to results very close to the basis set limit (see, e.g., Ref. 53). In order to avoid the problem of linear dependence due to diffuse functions usually experienced in solids (as in the present case), the calculations on solid $\mathrm{Ne}$, Ar, and $\mathrm{Kr}$ were done without the augmentation functions by using the cc-pV5Z basis sets. ${ }^{67,68}$ The face-centered cubic (fcc) structure was considered for the rare-gas solids and we checked that using a unit cell comprising 32 atoms $(2 \times 2 \times 2$ of the fcc four-atom unit cell) gives results which are very well converged with respect to the size of the supercell.

The nonlocal term [Eq. (2)] was implemented according to the scheme of Román-Pérez and Soler, ${ }^{69}$ which uses fast Fourier transforms, and therefore leads to calculations scaling as $\mathcal{O}(N \log N)$ ( $N$ is the number of points on the grid) instead of $\mathcal{O}\left(N^{2}\right)$ for a direct evaluation of Eq. (2) in real space. Note that the method of Román-Pérez and Soler also leads to an efficient calculation of the contribution of the nonlocal term to the KS-DFT potential (needed for the forces) and stress tensor. ${ }^{69,70}$ In our implementation, the nonlocal term is evaluated using only the smooth part of the electron density of the GAPW method. However, in Ref. 25, it was shown that within the projected-augmented wave ${ }^{71}$ method, plugin the all-electron density or the valence density into Eq. (2) leads to very similar results. Actually, we checked that our results agree very closely with the results obtained with other codes when available $\left[\mathrm{Ar}_{2}\right.$ with vdW-DF ${ }^{19,34,72,73}$ and $(\mathrm{r}) \mathrm{VV} 10^{34,36}$ and $\mathrm{Kr}_{2}$ with vdW-DF ${ }^{19,72,74}$ and VV10 $\left.{ }^{36}\right]$.

In addition to the functionals already introduced in Sec. I, we also mention rVV10, ${ }^{34}$ which is a revised version of $\mathrm{VV} 10^{36}$ such that its evaluation can also be done with the method of Román-Pérez and Soler. It was shown (Ref. 34) that VV10 and rVV10 give very similar results. rVV10 is among the functionals tested in the present work (Table I). For comparison purposes, we also considered the standard functionals LDA and PBE, ${ }^{5}$ as well as the dispersion-corrected functionals PBE-D3, revPBE-D3, and B97D-D3 (B97D ${ }^{15}$ is a reparametrization of $\mathrm{B} 97^{75}$ ), where $\mathrm{D} 3$ refers to the third set of parameters $C_{n}^{A B}$ [in Eq. (1)] proposed by Grimme (the three-body term was included in our calculations). ${ }^{18}$ Note that we used the VWN5 parametrization ${ }^{33}$ for the LDA correlation. Finally, we mention that LIBXC, a library of exchange-correlation functionals ${ }^{76}$ has been used for the evaluation of some of the semilocal functionals in Table I. 
TABLE II. Equilibrium bond length $R_{0}$ (in $\AA$ ) and interaction energy $\Delta E$ (in meV and with opposite sign) of rare-gas dimers calculated from various functionals and compared to accurate reference values and results obtained from the exchange-hole dipole moment model of Becke and Johnson (BJ).

\begin{tabular}{|c|c|c|c|c|c|c|c|c|}
\hline \multirow[b]{2}{*}{ Functional } & \multicolumn{2}{|c|}{$\mathrm{He}_{2}$} & \multicolumn{2}{|c|}{$\mathrm{Ne}_{2}$} & \multicolumn{2}{|c|}{$\mathrm{Ar}_{2}$} & \multicolumn{2}{|c|}{$\mathrm{Kr}_{2}$} \\
\hline & $R_{0}$ & $\Delta E$ & $R_{0}$ & $\Delta E$ & $R_{0}$ & $\Delta E$ & $R_{0}$ & $\Delta E$ \\
\hline LDA & 2.40 & 9.6 & 2.64 & 20.4 & 3.40 & 30.9 & 3.68 & 36.7 \\
\hline PBE & 2.76 & 3.2 & 3.08 & 5.6 & 4.00 & 6.3 & 4.36 & 6.9 \\
\hline vdW-DF & 2.82 & 6.6 & 3.07 & 14.1 & 3.92 & 23.1 & 4.27 & 26.2 \\
\hline vdW-DF2 & 2.75 & 2.8 & 2.95 & 9.2 & 3.75 & 18.3 & 4.09 & 22.3 \\
\hline $\mathrm{C} 09_{\mathrm{x}}-\mathrm{vdW}$ & 3.19 & 4.1 & 3.51 & 6.6 & 4.37 & 11.5 & 4.71 & 13.4 \\
\hline optB88-vdW & 3.48 & 0.5 & 3.30 & 3.0 & 3.93 & 11.7 & 4.20 & 16.1 \\
\hline $\mathrm{RPBEc} 2 / 3+\mathrm{nl}$ & 2.66 & 11.2 & 2.93 & 23.1 & 3.77 & 34.4 & 4.10 & 38.0 \\
\hline rVV10 & 2.92 & 0.9 & 3.01 & 5.6 & 3.73 & 13.9 & 4.00 & 19.3 \\
\hline PBE-D3 & 2.66 & 5.7 & 3.01 & 9.9 & 3.88 & 15.3 & 4.16 & 19.3 \\
\hline revPBE-D3 & 2.90 & 3.0 & 3.20 & 5.6 & 3.93 & 12.8 & 4.18 & 17.9 \\
\hline B97D-D3 & 3.01 & 2.4 & 3.33 & 4.3 & 3.99 & 11.3 & 4.18 & 17.2 \\
\hline PW86xPBEc-BJ ${ }^{a}$ & 3.01 & 0.8 & 3.12 & 3.8 & 3.84 & 11.2 & 4.07 & 17.0 \\
\hline Reference $^{\mathrm{b}}$ & 2.97 & 0.9 & 3.09 & 3.6 & 3.76 & 12.4 & 4.01 & 17.4 \\
\hline
\end{tabular}

\footnotetext{
${ }^{\text {a }}$ Reference 50.

b Reference 77 .
}

\section{RESULTS}

\section{A. Rare-gas dimers}

The interaction energy curves of the rare-gas dimers $\mathrm{He}_{2}, \mathrm{Ne}_{2}, \mathrm{Ar}_{2}$, and $\mathrm{Kr}_{2}$ are displayed in Fig. 1 and the corresponding values at the minimum (equilibrium distance $R_{0}$ and binding energy $\Delta E$ ) are shown in Table I. The KS-DFT results are compared to very accurate reference (theoretical or experimental, see Ref. 77 for details) results.

Discussing first the results obtained with the (semi)local functionals, it is already known ${ }^{7,8}$ 

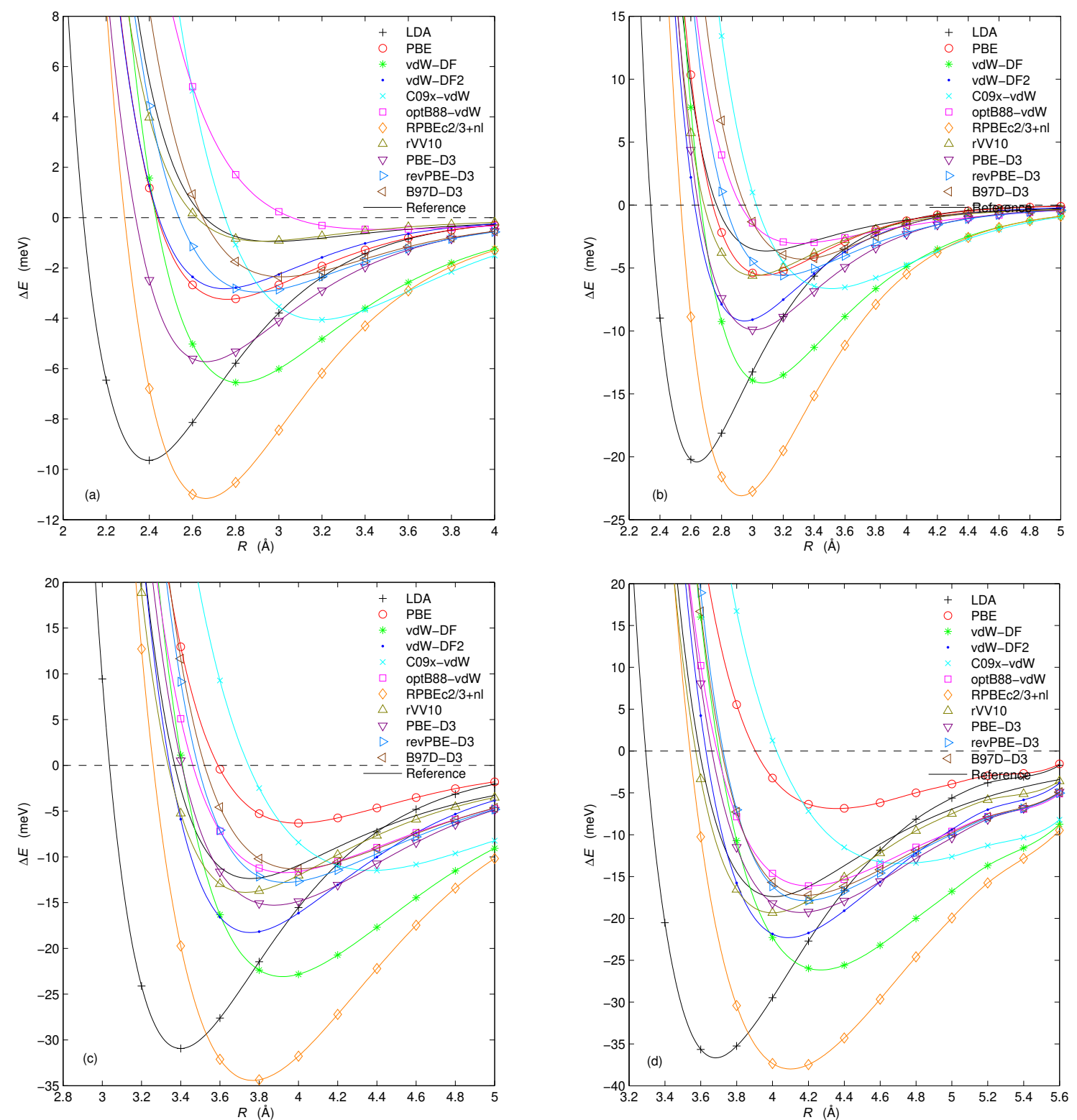

FIG. 1. Interaction energy curves for (a) $\mathrm{He}_{2}$, (b) $\mathrm{Ne}_{2}$, (c) $\mathrm{Ar}_{2}$, and (d) $\mathrm{Kr}_{2}$ obtained from various functionals and compared to reference results ${ }^{77}$ (black line without symbols).

that LDA strongly underestimates the bond lengths and overestimates the binding energies of all dimers, this trend being systematically observed with LDA for intermolecular complexes. For $\mathrm{He}_{2}$ and $\mathrm{Ne}_{2}$, LDA leads to binding energies which are one order of magnitude too large and to distances which are about $0.5 \AA$ too small. Among the countless semilocal and hybrid functionals tested on rare-gas dimers, PBE (a GGA free of any empirical parameter) is one of the most accurate (or least inaccurate, see Refs. 44, 45, and 47 for extensive tests). Still, 
PBE accuracy can not be considered as satisfying, $\mathrm{Ne}_{2}$ excepted, since it largely overbinds $\mathrm{He}_{2}$ and underbinds $\mathrm{Ar}_{2}$ and $\mathrm{Kr}_{2}$. In the group of semilocal and hybrid functionals (which do not include the physics of dispersion interactions), it was shown ${ }^{47}$ that the hybrid B97$1^{78}$ and meta-GGA hybrid M05-2X ${ }^{79}$ are also among the best for rare-gas dimers, but as in the case of PBE the results are in some cases rather inaccurate. Finally, from all previous studies on rare-gas dimers, we can conclude that there is no semilocal or hybrid functional that can be considered as reliable.

Turning now to the results obtained with the six nonlocal functionals, we can see that a large range of results can be obtained. vdW-DF largely overbinds all four dimers, and while the bond length is reasonable for $\mathrm{He}_{2}$ and $\mathrm{Ne}_{2}$, it is too large by $0.2-0.3 \AA$ for $\mathrm{Ar}_{2}$ and $\mathrm{Kr}_{2}$. vdW-DF2 improves over vdW-DF for $\Delta E$ by reducing the overbinding by a factor of two, but now the bond lengths of $\mathrm{He}_{2}$ and $\mathrm{Ne}_{2}$ are clearly too short. The bond lengths obtained with the $\mathrm{C} 09_{\mathrm{x}} \mathrm{-vdW}$ functional are as inaccurate as the LDA ones, but with the opposite trend (overestimation ranging from $0.2 \AA$ for $\mathrm{He}_{2}$ to $0.7 \AA$ for $\mathrm{Kr}_{2}$ ). The $\mathrm{C}_{0} 9_{\mathrm{x}^{-}}$ vdW interaction energies are not particularly accurate except for $\mathrm{Ar}_{2}$. optB88-vdW leads to quite accurate results for the binding energy $\Delta E$, however, the bond lengths $R_{0}$ are too large (in particular for $\mathrm{He}_{2}$ with $0.5 \AA$ of error). The binding energies obtained with the RPBEc2 $/ 3+$ nl functional constitute a disaster since they are even larger than LDA values, while the equilibrium bond length is accurate for $\mathrm{Ar}_{2}$ and $\mathrm{Kr}_{2}$, but not for the two lighter dimers. Among all tested functionals in this work, rVV10 is clearly the most accurate one. From Fig. 1(a), we can see that for $\mathrm{He}_{2}$ the rVV10 and reference curves coincide very closely along the whole range of considered intermolecular distances and correspond to the same binding energy $(0.9 \mathrm{meV})$. For the other dimers, also both the bond lengths and interaction energies are very accurate. The largest error in $\Delta E$ is only $2 \mathrm{meV}$ (for $\mathrm{Ne}_{2}$ and $\mathrm{Kr}_{2}$ ).

Concerning the three DFT-D3 methods that we considered, revPBE-D3 leads to a rather accurate bond length for $\mathrm{He}_{2}$, but overestimates $\Delta E$. For the other three dimers, revPBED3 yields values for $R_{0}$, which are too large by $0.1-0.2 \AA$, but quite accurate values for $\Delta E$. Overall, PBE-D3 leads to values which are less satisfying than revPBE-D3. B97D-D3 leads to results which are quite similar to revPBE-D3, but overestimates the bond lengths even more for $\mathrm{Ne}_{2}$ and $\mathrm{Ar}_{2}$. When compared to the nonlocal functionals, revPBE-D3 and B97D-D3 seem to show more stability in the results, except when compared to rVV10 which is by far the most accurate functional. 

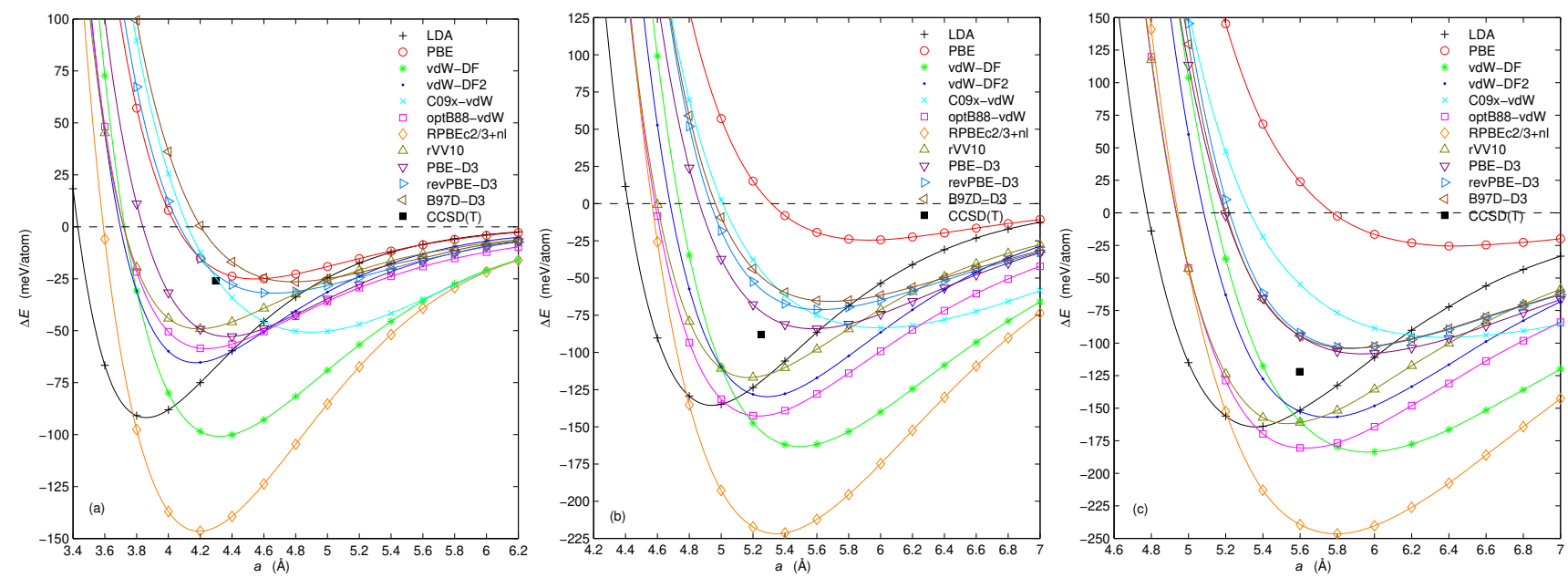

FIG. 2. Cohesive energy curves for (a) Ne, (b) Ar, and (c) Kr obtained from various functionals and compared to $\operatorname{CCSD}(\mathrm{T})$ results $^{81}$ (black square).

Among the previously published works on the testing of DFT functionals on rare-gas dimers, we should mention the results obtained by Kannemann and Becke ${ }^{50}$ with the functional PW86xPBEc-BJ (results also shown in Table II), where BJ refers to the XDM model for dispersion of Becke and Johnson. ${ }^{16}$ Their calculated bond lengths and binding energies are in very close agreement with the reference results, and actually the accuracy of rVV10 and PW86xPBEc-BJ can be considered as similar. However, it is important to note that the two adjustable parameters in the PW86xPBEc-BJ functional were determined by minimizing the error for $\Delta E$ of a set of ten rare-gas dimers (all combinations involving $\mathrm{He}, \mathrm{Ne}$, Ar, and Kr). rVV10 (VV10) also contains two parameters, but one of them was adjusted such that the mean error of $C_{6}^{A A}$ coefficients for a set of 54 species (among them $\mathrm{He}, \mathrm{Ne}, \mathrm{Ar}$, and $\mathrm{Kr}$ ) is minimized, ${ }^{36}$ while the other was determined using the S22 set of noncovalent complexes, ${ }^{80}$ which does not contain any rare-gas atoms. Therefore, (r)VV10 was certainly not adjusted exclusively on rare-gas systems, which makes its excellent performances on these systems even more impressive. 
TABLE III. Equilibrium lattice constant $a_{0}$ (in $\AA$ ) and cohesive energy $\Delta E$ (in $\mathrm{meV} /$ atom and with opposite sign) of rare-gas solids calculated from various functionals and compared to reference $[\mathrm{CCSD}(\mathrm{T})]$ results as well as the PBE-TS and RPA methods.

\begin{tabular}{|c|c|c|c|c|c|c|}
\hline \multirow[b]{2}{*}{ Functional } & \multicolumn{2}{|c|}{$\mathrm{Ne}$} & \multicolumn{2}{|c|}{$\mathrm{Ar}$} & \multicolumn{2}{|c|}{$\mathrm{Kr}$} \\
\hline & $a_{0}$ & $\Delta E$ & $a_{0}$ & $\Delta E$ & $a_{0}$ & $\Delta E$ \\
\hline LDA & 3.86 & 92 & 4.94 & 136 & 5.36 & 164 \\
\hline $\mathrm{PBE}$ & 4.55 & 25 & 5.93 & 25 & 6.42 & 25 \\
\hline vdW-DF & 4.32 & 101 & 5.49 & 163 & 5.96 & 184 \\
\hline vdW-DF2 & 4.17 & 65 & 5.29 & 130 & 5.75 & 157 \\
\hline $\mathrm{C} 09_{\mathrm{x}}-\mathrm{vdW}$ & 4.90 & 51 & 6.00 & 83 & 6.39 & 96 \\
\hline optB88-vdW & 4.24 & 59 & 5.24 & 143 & 5.63 & 181 \\
\hline $\mathrm{RPBEc} 2 / 3+\mathrm{nl}$ & 4.19 & 146 & 5.35 & 222 & 5.80 & 246 \\
\hline rVV10 & 4.19 & 49 & 5.17 & 117 & 5.53 & 162 \\
\hline PBE-D3 & 4.37 & 53 & 5.58 & 84 & 5.93 & 108 \\
\hline revPBE-D3 & 4.66 & 32 & 5.62 & 71 & 5.89 & 104 \\
\hline B97D-D3 & 4.78 & 26 & 5.69 & 66 & 5.87 & 104 \\
\hline PBE-TS ${ }^{\mathrm{a}}$ & 4.42 & 43 & 5.51 & 83 & 5.90 & 97 \\
\hline $\mathrm{RPA}(\mathrm{PBE})^{\mathrm{b}}$ & 4.5 & 17 & 5.3 & 83 & 5.7 & 112 \\
\hline $\operatorname{CCSD}(\mathrm{T})^{\mathrm{c}}$ & 4.297 & 26 & 5.251 & 88 & 5.598 & 122 \\
\hline
\end{tabular}

\footnotetext{
${ }^{\text {a }}$ Reference 61.

${ }^{\mathrm{b}}$ RPA energy evaluated with PBE orbitals and eigenvalues. ${ }^{56}$

${ }^{\mathrm{c}}$ Reference 81.
}

\section{B. Rare-gas solids}

The results for the rare-gas solids Ne, Ar, and Kr are shown in Fig. 2 and Table III. The reference results were obtained from coupled cluster with single, double, and perturbative triple excitations $[\mathrm{CCSD}(\mathrm{T})]$ calculations. ${ }^{81}$ For a meaningful comparison of our KS-DFT results with the $\operatorname{CCSD}(\mathrm{T})$ results, the zero-point energy calculated in Ref. 81 has been removed from the $\operatorname{CCSD}(\mathrm{T})$ results.

As for the dimers, LDA leads to severe underestimation of the lattice constant $a_{0}$ and 
overestimation of the cohesive energy $\Delta E$ for all three solids. Note, however, that in some cases LDA can, at a qualitative level, give relatively correct results for solids which are bound by weak interactions. Such examples include layered solids like graphite (see, e.g., Refs. 82 and 55). As already observed in Ref. 56, PBE gives essentially the same cohesive energy (25 meV/atom) for the three solids (very large underestimation for Ar and Kr). This is somewhat similar to what is observed for the corresponding dimers (see Table II). The PBE lattice constants are by far too large (by more than $0.7 \AA$ for Ar and Kr).

Concerning the nonlocal functionals based on the DRSLL or LMKLL kernels, the obser-

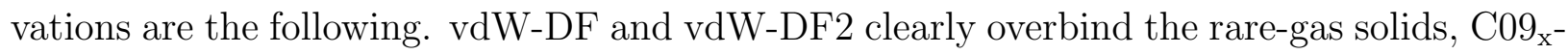
vdW totally fails for the lattice constant, and RPBEc $2 / 3+$ nl leads to the largest overbinding (as for the dimers). optB88-vdW leads to quite accurate values for $a_{0}$, but overestimates the cohesive energy rather strongly $(50 \%-100 \%)$, while in the case of the dimers optB88-vdW was quite good for the interaction energy.

The rVV10 nonlocal functional seems to be again superior to the other nonlocal functionals. The bond lengths are rather good (albeit too short by $\sim 0.1 \AA$ for Ne), while the cohesive energies are too large for all three solids, but the error is smaller than for the other nonlocal functionals except $\mathrm{C} 09_{\mathrm{x}}-\mathrm{vdW}$. The cohesive energies obtained with the DFT-D3 methods are quite accurate (PBE-D3 for Ne excepted), however the lattice constants are consistently too large by more than $0.3 \AA$ in most cases.

Also shown in Table II are the results from Ref. 61 obtained with the Tkatchenko and Scheffler $^{17}$ (TS) approach using PBE for the semilocal part. We can see that the PBE-TS results are similar to the results from PBE-D3 for both the lattice constant and cohesive energy. For completeness, we also show in Table II the values from the non-DFT method RPA (random-phase approximation). ${ }^{56}$ The RPA bond lengths are somehow overestimated, but the cohesive energies are very close to the $\operatorname{CCSD}(\mathrm{T})$ values. The RPA method is superior to the KS-DFT methods considered in the present work, but leads to calculations which are obviously much more expensive. Finally, we mention the DFT+XDM results from Ref. 62 for the lattice constant $a_{0}$, where the XDM dispersion correction was added to two different GGA functionals. The results are good only for Kr, whereas for Ne and Ar rather inaccurate values were obtained.

As a summary of the results on rare-gas solids, the rVV10 nonlocal functional seems to be a relatively good choice (at least compared to the other functionals), but leads to 
non-negligible overestimations of the cohesive energy, and this more than in the case of the rare-gas dimers.

\section{FURTHER DISCUSSION}

\section{A. Energy decomposition}

In order to have more insight into the results of Secs. III A and III B, we now consider the various contributions to the interaction energy $\Delta E$ and their relative importance. Figure 3 shows for the Ar dimer (similar trends are observed for the other rare-gas dimers and solids) the contributions to $\Delta E$ coming from the (semi)local exchange-correlation functional $\left(\Delta E_{\mathrm{xc}}^{\mathrm{sl}}\right)$, the (atom-pairwise or nonlocal) dispersion energy term $\left(\Delta E_{\mathrm{c}}^{\mathrm{disp}}\right)$, the sum of these two $\left(\Delta E_{\mathrm{xc}}=\Delta E_{\mathrm{xc}}^{\mathrm{sl}}+\Delta E_{\mathrm{c}}^{\mathrm{disp}}\right)$, the rest of the terms $\left(\Delta E_{\text {rest }}\right)$ representing the kinetic and electrostatic energies, and the sum $\Delta E_{\text {rest }}+\Delta E_{\mathrm{xc}}^{\mathrm{sl}}$. The sum of $\Delta E_{\mathrm{xc}}$ and $\Delta E_{\mathrm{rest}}$ gives the total interaction energy $\Delta E$ shown in Fig. 1(c).

Around the experimental equilibrium bond length $(\sim 3.8 \AA)$ all terms seem to be of roughly equal importance and the absolute values range from 10 to $50 \mathrm{meV}$ depending on the functional. However, for smaller bond lengths $R$, the curves $\Delta E_{\mathrm{xc}}^{\mathrm{sl}}$ and $\Delta E_{\mathrm{rest}}$ vary faster than $\Delta E_{\mathrm{c}}^{\text {disp }}$ and these terms become much more important. For instance, at $R=2.0 \AA$ (not shown), the magnitude of $\Delta E_{\mathrm{rest}}$ and $\Delta E_{\mathrm{xc}}^{\mathrm{sl}}$ is around 8000 and $4000 \mathrm{meV}$, respectively, while for $\Delta E_{\mathrm{c}}^{\text {disp }}$ it is smaller than $5 \mathrm{meV}$ for the DFT-D3 methods and between 100 and $300 \mathrm{meV}$ for the nonlocal methods. Note that the different behavior of $\Delta E_{\mathrm{c}}^{\mathrm{disp}}$ for the DFT-D3 method

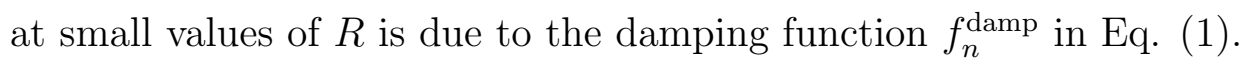

In Fig. 3(b), we can see that among the nonlocal functionals, rVV10 and DRSLL lead to the smallest and largest values (in magnitude) for $\Delta E_{\mathrm{c}}^{\mathrm{disp}}$, respectively. In the range of intermolecular distances that we considered, $\Delta E_{\mathrm{c}}^{\text {disp }}$ is negative, however, the energy (the component of the total energy) is always positive in the case of the nonlocal functionals [Eq. (2)], while the values are negative for the atom-pairwise DFT-D3 method [Eq. (1)].

The sum of the terms $\Delta E_{\mathrm{xc}}^{\mathrm{sl}}$ and $\Delta E_{\mathrm{rest}}$, which represents the interaction energy of $\mathrm{Ar}_{2}$ calculated without the dispersion term, is shown in Fig. 3(e). Among the semilocal functionals only PBE and RPBEc2/3 yield reasonable interaction energies, while all other functionals, except LDA barely bind or do not bind at all the two Ar atoms. Actually, it is clear that 

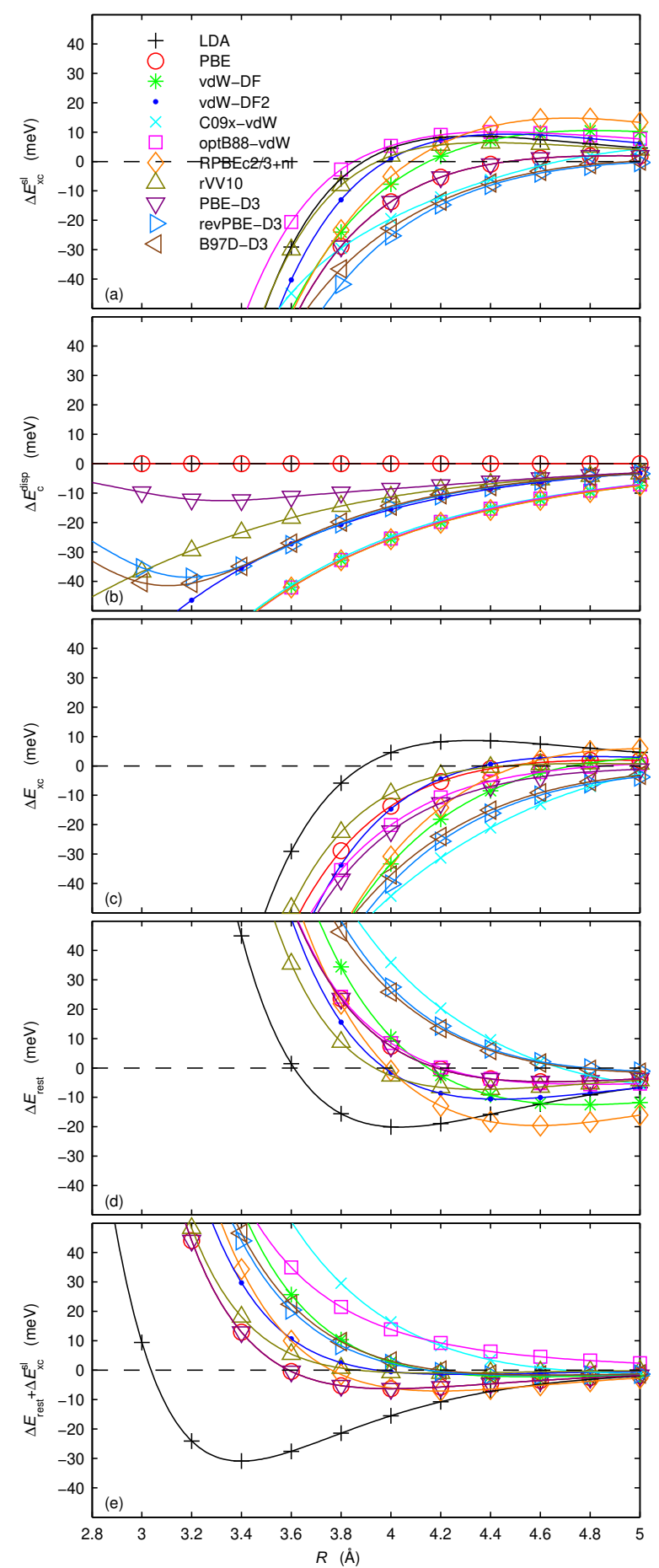

FIG. 3. Contributions to the $\mathrm{Ar}_{2}$ interaction energy coming from (a) the semilocal (sl) xc functional, (b) the dispersion energy [Eqs. (1) or (2)], (c) the total (sl plus dispersion) xc functional, (d) the rest (kinetic plus electrostatic), and (e) the sum of sl and rest. The addition of (c) and (d) gives the total interaction energy of Fig. 1(c). 
in order to avoid overbinding (due to double counting) when using a dispersion term in the total-energy expression, it should be combined with a semilocal functional which leads to strongly underestimated interaction energy. ${ }^{19}$

From Fig. 3, we can also infer that the differences in $\Delta E$ between the functionals can not be understood by looking exclusively at the contribution from the exchange-correlation energy. Indeed, the curves for $\Delta E_{\text {rest }}$ (whose analytical form is the same for all functionals) show differences which are as strong as for $\Delta E_{\mathrm{xc}}^{\mathrm{sl}}$ and $\Delta E_{\mathrm{c}}^{\mathrm{disp}}$. Actually, the differences in the $\Delta E_{\text {rest }}$ curves are a reflection of the corresponding exchange-correlation potentials $v_{\mathrm{xc}}=$ $\delta E_{\mathrm{xc}} / \delta \rho$ used in the KS-DFT equations. It is known that for the calculation of properties depending on total energies, the results usually do not depend too sensitively on the orbitals and electron density plugged into the total-energy functional (but one needs to be very careful with this statement). However, the individual components of the total energy show much stronger sensitivity, but these variations tend to cancel among the different terms. For $\mathrm{Ar}_{2}$, we also performed non-self-consistent calculations by evaluating all functionals with the PBE orbitals and electron density (results shown in Fig. 4). The resulting equilibrium bond lengths and binding energies are essentially the same as their self-consistent counterparts. However, from Fig. 4(a) we can see that for some functionals (LDA and C09 $-\mathrm{vdW}$ in

particular), the $\Delta E_{\mathrm{xc}}^{\mathrm{sl}}$ curve is quite different to the one obtained self-consistently [Fig. 3(a)]. For the nonlocal dispersion terms (DRSLL, LMKLL, and rVV10), basically no difference between the self-consistent and non-self-consistent calculations can be seen, which is maybe due to the fact that these terms are evaluated only with the smooth part of the electron density.

\section{B. Three-body interaction energy}

The leading term in the many-body contribution to the interaction energy is the threebody nonadditive energy $\Delta E_{3}$. In the case of a simple trimer this term is calculated as the atomization energy of the trimer minus the sum of the atomization energies of the three dimers. If the three atoms in the trimer are identical, then

$$
\Delta E_{3}=E_{\text {tot }}^{\text {trimer }}-3 E_{\text {tot }}^{\text {dimer }}+3 E_{\text {tot }}^{\text {monomer }}
$$


The asymptotic behavior of the dispersion component of $\Delta E_{3}$ is given by the Axilrod-TellerMuto $^{84,85}$ (ATM) triple-dipole term

$$
\Delta E_{3}^{\mathrm{ATM}}=C_{9}^{A B C} \frac{1+\cos \left(\theta_{A B C}\right) \cos \left(\theta_{B C A}\right) \cos \left(\theta_{C A B}\right)}{\left(R_{A B} R_{B C} R_{C A}\right)^{3}},
$$

where $\theta_{i j k}$ and $R_{i j}$ are the angles and side lengths of the triangle formed by the trimer and $C_{9}^{A B C}$ is the triple-dipole constant.

Using Eq. (4), we calculated $\Delta E_{3}$ for the Ar trimer at equilateral geometry, and in Fig. 5 the results are compared to the accurate $\operatorname{CCSD}(\mathrm{T})$ values from Ref. 83 as well as the asymptotic ATM term [Eq. (5)] with $C_{9}^{A B C}=521.7$ au. ${ }^{86}$ In general, the three-body interaction energy $\Delta E_{3}$ of a trimer in this configuration is the largest contribution to the many-body cohesive energy of the corresponding solid in the fcc structure. For interatomic distances $R$ larger than $\sim 3.5 \AA$, we can see that most functionals strongly overestimate (too positive values) the three-body energy $\Delta E_{3}$. The exceptions are optB88-vdW which leads to negative values for the whole range of interatomic distances $R$ that we considered and rVV10 which seems to be the best of the considered functionals. Closely around the equilibrium interatomic distances in the dimer and solid $(\sim 3.7-3.75 \AA)$, the rVV10 values are close to the $\operatorname{CCSD}(\mathrm{T})$ and $\mathrm{ATM}$ values. However, the maximum of the $\Delta E_{3}$ curve is at $\sim 3.2 \AA$ for rVV10 (and overestimated), while it is at $\sim 3.7 \AA$ for $\operatorname{CCSD}(\mathrm{T})$ ). In Refs. 57 and 59 , other functionals were considered for the calculation of $\Delta E_{3}$, but none of them lead to results in qualitative agreement with the $\operatorname{CCSD}(\mathrm{T})$ results.

\section{SUMMARY}

We have presented the results of KS-DFT calculations on rare-gas dimers and solids. The focus was on the performance of nonlocal vdW functionals for the equilibrium bond length and binding energy. The main conclusions are (a) overall the rVV10 functional is the one performing the best, (b) some others (e.g., C09 $x$ or RPBEc $2 / 3+$ nl) can perform very badly, and (c) the considered DFT-D3 methods show good accuracy for the interaction energy, but seem to lead to some (slight) overestimation of the bond lengths. In Sec. IV A, we presented an analysis by decomposing the interaction energy into its components in order to estimate the relative importance of each term, and in Sec. IV B it was shown that rVV10 gives also

reasonable values (at least close to the equilibrium geometry) for the three-body nonadditive energy, while the other DFT functionals are very inaccurate. 
Considering the results on rare-gas systems obtained in the present work and from previously published papers, the (r)VV10 nonlocal functional seems to be the most accurate among the DFT methods. It was already shown to be accurate for many other finite systems, ${ }^{37}$ while for layered solids it is necessary to modify its parameters (and eventually combine it with another semilocal functional) to get accurate results. ${ }^{39}$

\section{ACKNOWLEDGMENTS}

The research leading to these results has received funding from the Swiss University Conference through the High Performance and High Productivity Computing (HP2C) Programme. Computer resources were provided by the University of Zurich.

\section{REFERENCES}

${ }^{1}$ W. Kohn and L. J. Sham, Phys. Rev. 140, A1133 (1965).

${ }^{2}$ P. Hohenberg and W. Kohn, Phys. Rev. 136, B864 (1964).

${ }^{3}$ A. J. Cohen, P. Mori-Sánchez, and W. Yang, Chem. Rev. 112, 289 (2012).

${ }^{4}$ A. D. Becke, Phys. Rev. A 38, 3098 (1988).

${ }^{5}$ J. P. Perdew, K. Burke, and M. Ernzerhof, Phys. Rev. Lett. 77, 3865 (1996), 78, 1396 (1997).

${ }^{6}$ A. D. Becke, J. Chem. Phys. 98, 5648 (1993).

${ }^{7}$ S. Kristyán and P. Pulay, Chem. Phys. Lett. 229, 175 (1994).

${ }^{8}$ J. M. Pérez-Jordá and A. D. Becke, Chem. Phys. Lett. 233, 134 (1995).

${ }^{9}$ E. R. Johnson, I. D. Mackie, and G. A. DiLabio, J. Phys. Org. Chem. 22, 1127 (2009).

${ }^{10}$ A. Tkatchenko, L. Romaner, O. T. Hofmann, E. Zojer, C. Ambrosch-Draxl, and M. Scheffler, MRS Bull. 35, 435 (2010).

${ }^{11}$ S. Grimme, WIREs Comput. Mol. Sci. 1, 211 (2011).

${ }^{12}$ J. Klimeš and A. Michaelides, J. Chem. Phys. 137, 120901 (2012).

${ }^{13}$ Q. Wu and W. Yang, J. Chem. Phys. 116, 515 (2002).

${ }^{14}$ S. Grimme, J. Comput. Chem. 25, 1463 (2004).

${ }^{15}$ S. Grimme, J. Comput. Chem. 27, 1787 (2006).

${ }^{16}$ A. D. Becke and E. R. Johnson, J. Chem. Phys. 122, 154104 (2005). 
${ }^{17}$ A. Tkatchenko and M. Scheffler, Phys. Rev. Lett. 102, 073005 (2009).

${ }^{18}$ S. Grimme, J. Antony, S. Ehrlich, and H. Krieg, J. Chem. Phys. 132, 154104 (2010).

${ }^{19}$ M. Dion, H. Rydberg, E. Schröder, D. C. Langreth, and B. I. Lundqvist, Phys. Rev. Lett. 92, 246401 (2004), 95, 109902 (2005).

${ }^{20}$ D. C. Langreth and J. P. Perdew, Solid State Commun. 17, 1425 (1975).

${ }^{21}$ O. Gunnarsson and B. I. Lundqvist, Phys. Rev. B 13, 4274 (1976), 15, 6006 (1977).

${ }^{22}$ D. C. Langreth and J. P. Perdew, Phys. Rev. B 15, 2884 (1977).

${ }^{23}$ Y. Zhang and W. Yang, Phys. Rev. Lett. 80, 890 (1998).

${ }^{24}$ D. C. Langreth, B. I. Lundqvist, S. D. Chakarova-Käck, V. R. Cooper, M. Dion, P. Hyldgaard, A. Kelkkanen, J. Kleis, L. Kong, S. Li, P. G. Moses, E. Murray, A. Puzder, H. Rydberg, E. Schröder, and T. Thonhauser, J. Phys.: Condens. Matter 21, 084203 (2009).

${ }^{25}$ J. Klimeš, D. R. Bowler, and A. Michaelides, Phys. Rev. B 83, 195131 (2011).

${ }^{26}$ K. Lee, E. D. Murray, L. Kong, B. I. Lundqvist, and D. C. Langreth, Phys. Rev. B 82, 081101(R) (2010).

${ }^{27}$ E. D. Murray, K. Lee, and D. C. Langreth, J. Chem. Theory Comput. 5, 2754 (2009).

${ }^{28}$ J. P. Perdew and Y. Wang, Phys. Rev. B 33, 8800 (1986).

${ }^{29}$ V. R. Cooper, Phys. Rev. B 81, 161104(R) (2010).

${ }^{30}$ J. Klimeš, D. R. Bowler, and A. Michaelides, J. Phys.: Condens. Matter 22, 022201 (2010).

${ }^{31}$ J. Wellendorff and T. Bligaard, Top. Catal. 54, 1143 (2011).

${ }^{32}$ B. Hammer, L. B. Hansen, and J. K. Nørskov, Phys. Rev. B 59, 7413 (1999).

${ }^{33}$ S. H. Vosko, L. Wilk, and M. Nusair, Can. J. Phys. 58, 1200 (1980).

${ }^{34}$ R. Sabatini, T. Gorni, and S. de Gironcoli, Phys. Rev. B 87, 041108(R) (2013).

${ }^{35}$ O. A. Vydrov and T. Van Voorhis, Phys. Rev. Lett. 103, 063004 (2009).

${ }^{36}$ O. A. Vydrov and T. Van Voorhis, J. Chem. Phys. 133, 244103 (2010).

${ }^{37}$ W. Hujo and S. Grimme, J. Chem. Theory Comput. 7, 3866 (2011).

${ }^{38}$ T. Björkman, A. Gulans, A. V. Krasheninnikov, and R. M. Nieminen, J. Phys.: Condens. Matter 24, 424218 (2012).

${ }^{39}$ T. Björkman, Phys. Rev. B 86, 165109 (2012).

${ }^{40}$ D. C. Patton and M. R. Pederson, Phys. Rev. A 56, R2495 (1997), 71, 019906(E) (2005).

${ }^{41}$ D. C. Patton and M. R. Pederson, Int. J. Quantum Chem. 69, 619 (1998). 
${ }^{42}$ T. van Mourik and R. J. Gdanitz, J. Chem. Phys. 116, 9620 (2002).

${ }^{43} \mathrm{X} . \mathrm{Xu}$ and W. A. Goddard III, Proc. Natl. Acad. Sci. U.S.A. 101, 2673 (2004).

${ }^{44}$ E. R. Johnson, R. A. Wolkow, and G. A. DiLabio, Chem. Phys. Lett. 394, 334 (2004).

${ }^{45} \mathrm{X} . \mathrm{Xu}, \mathrm{Q}$. Zhang, R. P. Muller, and W. A. Goddard III, J. Chem. Phys. 122, 014105 (2005).

${ }^{46}$ J. Tao and J. P. Perdew, J. Chem. Phys. 122, 114102 (2005).

${ }^{47}$ Y. Zhao and D. G. Truhlar, J. Phys. Chem. A 110, 5121 (2006).

${ }^{48}$ I. C. Gerber and J. G. Ángyán, J. Chem. Phys. 126, 044103 (2007).

${ }^{49}$ G. Murdachaew, S. de Gironcoli, and G. Scoles, J. Phys. Chem. A 112, 9993 (2008).

${ }^{50}$ F. O. Kannemann and A. D. Becke, J. Chem. Theory Comput. 5, 719 (2009).

${ }^{51}$ E. R. Johnson, A. D. Becke, C. D. Sherrill, and G. A. DiLabio, J. Chem. Phys. 131, 034111 (2009).

${ }^{52}$ K. E. Yousaf and E. N. Brothers, J. Chem. Theory Comput. 6, 864 (2010).

${ }^{53}$ D. Roy, M. Marianski, N. T. Maitra, and J. J. Dannenberg, J. Chem. Phys. 137, 134109 (2012).

${ }^{54}$ F. Ortmann, F. Bechstedt, and W. G. Schmidt, Phys. Rev. B 73, 205101 (2006).

${ }^{55}$ F. Tran, R. Laskowski, P. Blaha, and K. Schwarz, Phys. Rev. B 75, 115131 (2007).

${ }^{56}$ J. Harl and G. Kresse, Phys. Rev. B 77, 045136 (2008).

${ }^{57}$ A. Tkatchenko and O. A. von Lilienfeld, Phys. Rev. B 78, 045116 (2008).

${ }^{58}$ P. Haas, F. Tran, and P. Blaha, Phys. Rev. B 79, 085104 (2009), 79, 209902(E) (2009).

${ }^{59}$ K. A. Maerzke, G. Murdachaew, C. J. Mundy, G. K. Schenter, and J. I. Siepmann, J. Phys. Chem. A 113, 2075 (2009).

${ }^{60}$ T. Bučko, J. Hafner, S. Lebègue, and J. G. Ángyán, J. Phys. Chem. A 114, 11814 (2010).

${ }^{61}$ W. A. Al-Saidi, V. K. Voora, and K. D. Jordan, J. Chem. Theory Comput. 8, 1503 (2012).

${ }^{62}$ A. Otero-de-la Roza and E. R. Johnson, J. Chem. Phys. 136, 174109 (2012).

${ }^{63}$ J. VandeVondele, M. Krack, F. Mohamed, M. Parrinello, T. Chassaing, and J. Hutter, Comput. Phys. Commun. 167, 103 (2005).

${ }^{64} \mathrm{CP} 2 \mathrm{~K}$ version 2.4 . CP2K is freely available from www.cp2k.org.

${ }^{65}$ G. Lippert, J. Hutter, and M. Parrinello, Mol. Phys. 92, 477 (1997).

${ }^{66}$ G. Lippert, J. Hutter, and M. Parrinello, Theor. Chem. Acc. 103, 124 (1999).

${ }^{67}$ D. E. Woon and T. H. Dunning Jr., J. Chem. Phys. 100, 2975 (1994).

${ }^{68}$ A. K. Wilson, D. E. Woon, K. A. Peterson, and T. H. Dunning Jr., J. Chem. Phys. 110, 
7667 (1999).

${ }^{69}$ G. Román-Pérez and J. M. Soler, Phys. Rev. Lett. 103, 096102 (2009).

${ }^{70}$ R. Sabatini, E. Küçükbenli, B. Kolb, T. Thonhauser, and S. de Gironcoli, J. Phys.: Condens. Matter 24, 424209 (2012).

${ }^{71}$ P. E. Blöchl, Phys. Rev. B 50, 17953 (1994).

${ }^{72}$ T. Thonhauser, V. R. Cooper, S. Li, A. Puzder, P. Hyldgaard, and D. C. Langreth, Phys. Rev. B 76, 125112 (2007).

${ }^{73}$ D. Nabok, P. Puschnig, and C. Ambrosch-Draxl, Comput. Phys. Commun. 182, 1657 (2011).

${ }^{74}$ P. Lazić, N. Atodiresei, M. Alaei, V. Caciuc, S. Blügel, and R. Brako, Comput. Phys. Commun. 181, 371 (2010).

${ }^{75}$ A. D. Becke, J. Chem. Phys. 107, 8554 (1997).

${ }^{76}$ M. A. L. Marques, M. J. T. Oliveira, and T. Burnus, Comput. Phys. Commun. 183, 2272 (2012).

${ }^{77}$ K. T. Tang and J. P. Toennies, J. Chem. Phys. 118, 4976 (2003).

${ }^{78}$ F. A. Hamprecht, A. J. Cohen, D. J. Tozer, and N. C. Handy, J. Chem. Phys. 109, 6264 (1998).

${ }^{79}$ Y. Zhao, N. E. Schultz, and D. G. Truhlar, J. Chem. Theory Comput. 2, 364 (2006).

${ }^{80}$ P. Jurečka, J. Šponer, J. Černý, and P. Hobza, Phys. Chem. Chem. Phys. 8, 1985 (2006).

${ }^{81}$ K. Rościszewski, B. Paulus, P. Fulde, and H. Stoll, Phys. Rev. B 62, 5482 (2000).

${ }^{82}$ M. Hasegawa and K. Nishidate, Phys. Rev. B 70, 205431 (2004).

${ }^{83}$ R. Podeszwa and K. Szalewicz, J. Chem. Phys. 126, 194101 (2007).

${ }^{84}$ B. M. Axilrod and E. Teller, J. Chem. Phys. 11, 299 (1943).

${ }^{85}$ J. Muto, Proc. Phys. Math. Soc. Jpn. 17, 629 (1943).

${ }^{86}$ A. J. Thakkar, H. Hettema, and P. E. S. Wormer, J. Chem. Phys. 97, 3252 (1992). 

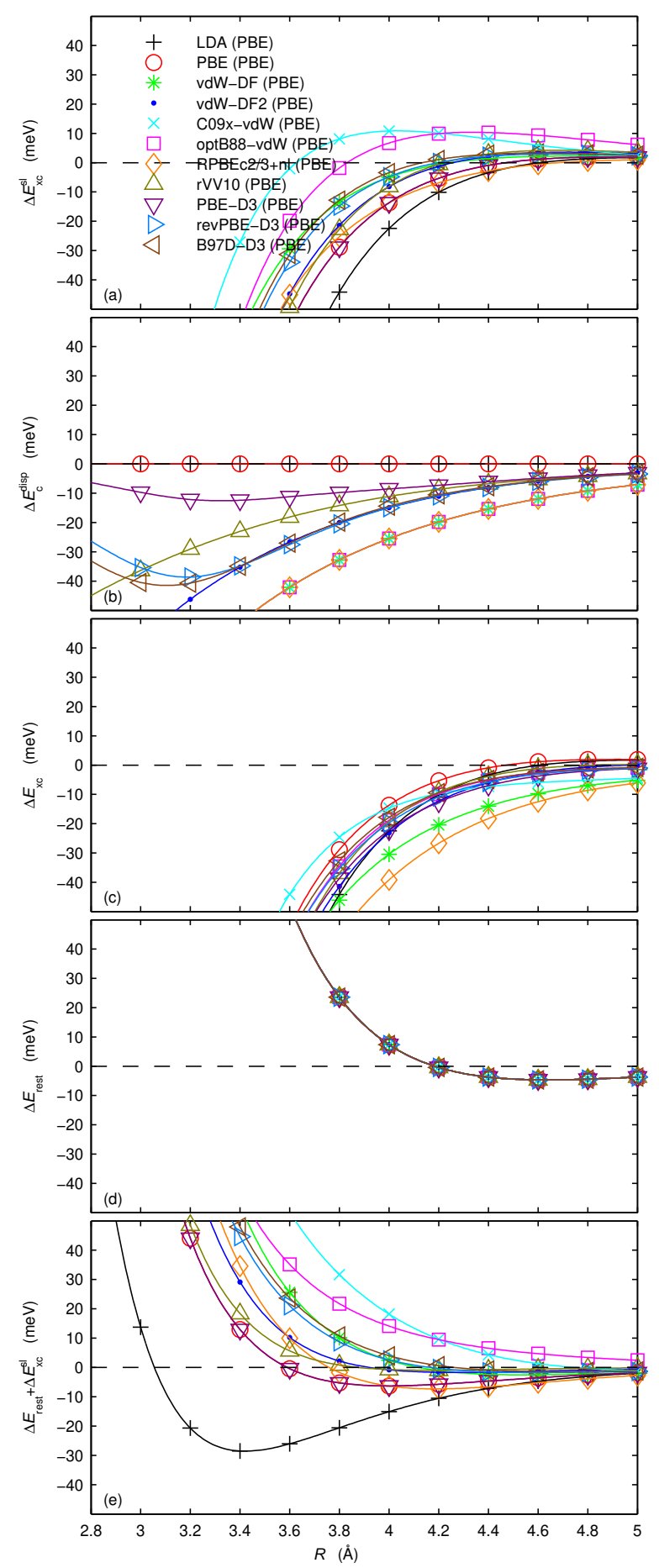

FIG. 4. Same as Fig. 3, but obtained from non-self-consistent calculations by plugin the PBE orbitals and electron density into the functionals. 


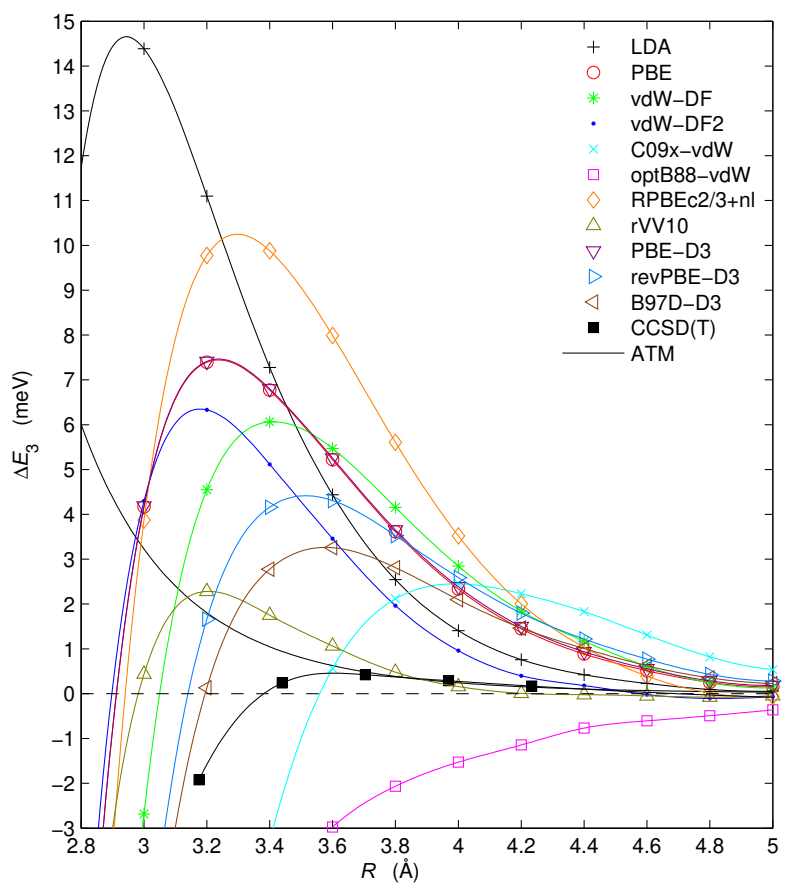

FIG. 5. Three-body interaction energy of $\mathrm{Ar}_{3}$ (equilateral triangular configuration) plotted against the Ar-Ar distance. The CCSD(T) results are from Ref. 83. ATM (black line without symbols) is the Axilrod-Teller-Muto term given by Eq. (5). 